\title{
Golf Club Deflection Characteristics as a Function of the Swing Hub Path
}

\author{
Ryan S. McGinnis and Steven Nesbit*
}

Department of Mechanical Engineering, Lafayette College, Easton, PA, USA

\begin{abstract}
This study investigated the relationships between golfer hub path trajectories and interaction kinetics, and club behavior. An equation of motion describing a flexible golf club system was derived and solved to yield time and club position deflection behavior during the downswing. This equation was applied to three diverse subjects whose kinematic and kinetic information was used to drive the simulation. It was determined that there is a relationship between the timing of the maximum interaction torque and the increase in normal force applied to the club and club head deflections. Also, it appears that there is a correlation between degree of radius reduction directly before impact and shaft deflection behavior. The timing of both torque and normal force are directly related to changes in hub path radius thus the effect of hub path geometry on club deflection behavior is secondary. Based upon these findings, a method for fitting shafts to specific swing characteristics was developed that optimized predicted carry distance. These results are based upon limited subjects.
\end{abstract}

Keywords: Golf swing, shaft deflection, swing hub, computer modeling.

\section{INTRODUCTION}

Golf is one of the most popular recreational sports with more than 55 million participants worldwide submitting themselves to the arduous task of trying to get a small ball into a small hole that is a very long distance away [1]. To compound the issue, the golf swing is one of the most difficult and complex motions in sports [1]. It depends on the synergy of many separate movements; timing of the wrists, squaring of the club face, and angle of the swing plane just to name a few. On top of all of this, the swing doesn't line up well with the main joints of the body, as say the baseball swing lines up with the spine for example. Researching this motion has the potential to make the game of golf a little bit easier for everyone [1]. Hopefully this will translate into not only lower scores, but also fewer golf-related injuries.

The shaft is the means by which energy is transferred from the golfer to the ball and as such, is an important of the golf club worthy of scientific study. Accordingly, there have been a number of studies conducted investigating the deflections experienced by the club during the downswing [2,3]. Milne and Davis [2] detailed the development of a computer simulation that modeled shaft deflection during the downswing of the golf shot as well as a method for collecting applied force and torque data using foil strain gauges. Both of these investigations utilized the double pendulum as a means for modeling the golf swing.

\section{Double Pendulum Model}

The double pendulum model was initially proposed by Cochran and Stobbs [4] to study the biomechanics of the golf swing. This model consists of two rigid links that are driven by torques at their joints as seen in Fig. (1).

*Address correspondence to this author at the Department of Mechanical Engineering, Lafayette College, Easton, PA, USA; Tel: 610 - 330 - 5446; Fax: 610 - 330 - 5905; E-mail: nesbits@lafayette.edu

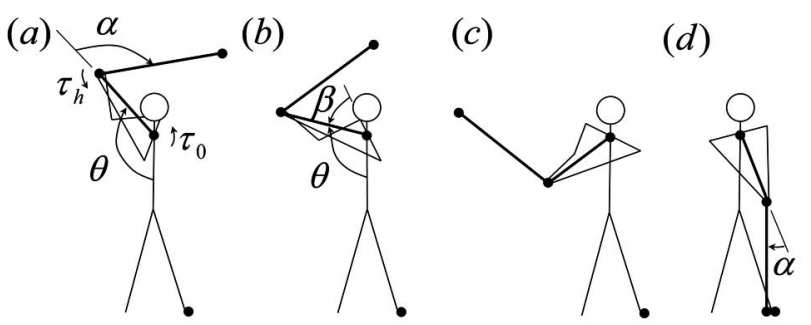

Fig. (1). Double Pendulum Model [5].

Cochran and Stobbs [4] constrained the swing to a constant radius hub path and a fixed center of curvature. This model has been used as the basis for many biomechanical studies of the golf swing because it was assumed to be reasonably accurate, it is very simple, and it ensures relative ease in computations.

In Milne and Davis' study [2], the bottom link, or club, was no longer constrained to be rigid. They modeled it as a flexible, slender beam with constant cross-section along its length that was driven by (strain gauge measured) shoulder and wrist torques. Their model predicted the following shaft behavior in the plane of the swing.

The results shown in Fig. (2), illustrate the several bending modes that occur during the downswing. The authors demarcate the swing as having three distinct phases based on the club deflection. The first phase is from 230 to $130 \mathrm{~ms}$ before impact, and is where the application of the wrist torque bends the shaft backwards. During the second phase (130 ms before until impact), the shaft gradually straightens and then bends forward due to the counterclockwise centrifugal torque that is applied to the lower end of the shaft. This torque is generated by the offset of the 
center of mass of the club head behind the centerline of the shaft. The third and final phase occurs at impact, where contact with the ball excites a few cycles of bending vibration [2]. This means that some of the energy at impact is spent exciting the shaft instead of being transferred to the ball. As a result, their model shows that a flexible shaft will produce an initial ball velocity about $4 \%$ slower than that produced by an idealized rigid shaft for the same input torques.

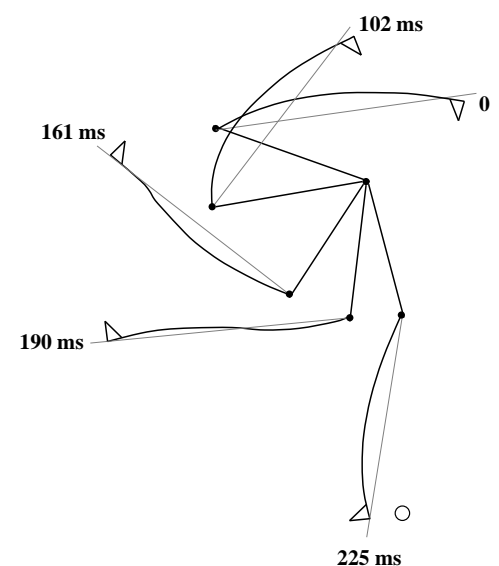

Fig. (2). Shaft deflections predicted by Milne and Davis [5].

The overall conclusion derived by Milne and Davis [2] is that the dynamic "springing back" of the shaft has little effect on ball velocity after impact. The main benefit resulting from a golf swing with a flexible shaft is an additional "dynamic loft" at impact. This means that if a driver initially has a loft of ten degrees (typical for most drivers), the bending mode experience by the club at impact would increase this loft to 20 or 30 degrees (measured from the vertical).

The work of Milne and Davis was generally supported by a number of studies that followed. Mather and Jowett [3] describe the same initial deflection followed by a "rebounding" of the club head. Penner [5] also supports the deflection progression during the downswing proposed by Milne and Davis. He proposes that if shaft flexibility is matched to a player's swing, the springing back of the shaft can help add to club head velocity at impact.

One of the goals of this thesis is to develop a shaft fitting method. Based on the observations of Milne and Davis [2] and Penner [5], a valuable shaft fitting method needs to match shaft flexibilities to specific golfers such that club head velocity and the dynamic loft of the club at impact are optimized to yield maximum ball carry.

\section{Hub Path Research Results}

Biomechanical studies of the golf swing, including that done by Milne and Davis [2], have predominantly assumed that the path of the hands through the swing, the hub path, has a fixed center of curvature and radius [1]. Studies have said that any deviations from this path are negligible and have little effect on required inputs or expected outputs [4]. Recent studies have shown however, that the path of the hands during the downswing is not circular, and that deviations from a circular hub path produce significant results with respect to the kinetic inputs required to produce a desired kinematic output (i.e. club head velocity) [1]. The non-circular nature of the hub path is clearly seen in the following figure.
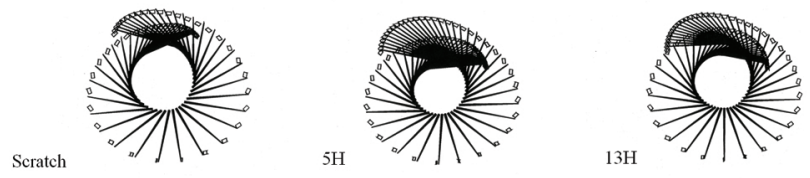

Fig. (3). subject swings [1].

The work of Nesbit and McGinnis [1] demonstrated a three phase pattern of the hub path which is based on path radius maximums and minimums. The first phase spans from the initial local radius maximum at $220 \mathrm{~ms}$ to the local minimum $120 \mathrm{~ms}$ before impact, this phase exhibits a radius reduction accompanied by gains in many of the swing quantities (i.e. club head velocity, torque, and force). The second phase extends from the local minimum at $120 \mathrm{~ms}$ to the next local maximum at $30 \mathrm{~ms}$ before impact. During this time, the radius increases and most of the swing quantities experience their most drastic increases as well. For example, the scratch golfer's club head velocity increases to $90 \%$ of its final value by the end of the second phase. The final phase spans the time from the last local maximum to impact. Again, we see a drastic radius reduction with many swing quantities reaching either their maximums or minimums at impact.

Since hub path affects the forces and torques applied to the club by the golfer, it is probable that it will also influence, through the kinetic inputs, the behavior of the club during the downswing. The relationships that exist between these inputs and the club behavior need to be understood so that shafts can be effectively "fit" to golfers.

It follows then that the goals of this thesis were to quantify the relationships between changes in hub path and the associated changes in shaft deflection. As well as to use this information and the information predicted by the solution to the equation of motion for a flexible club system to design a shaft fitting method that can fit shafts to golfers so as to optimize the carry distance of the golf ball.

\section{METHODS}

Motion capture data for three distinct subjects was used to develop the kinematic quantities that represent each of their swings. A rigid club model that does not restrict hand path to a circular arc was used to develop the kinetic inputs supplied by each golfer [1]. This force and moment data is then used to drive a partial differential equation that describes the deflection of the club. This equation was developed using the principles of Hamiltonian Dynamics. A solution to the partial differential equation is obtained using a numerical equation solver in Mathematica. A curve is fit to the stiffness data for the shaft (stiffness = modulus (E) * moment of inertia (I)) and is supplied for use by the solver. The damping term of the differential equation is adjusted until predicted shaft deflections match those found from empirical data. The deflection data is used to determine the dynamic loft and velocity of the club head at impact. These values allow for the development of a shaft fitting method that matches shaft stiffness to a player's swing. Deflection data is also compared with hub path radius and the kinetic 
quantities applied to the club to determine the relationships that exist between these values.

\section{DEFLECTION MODEL/SYSTEM EQUATION OF MOTION}

To determine the correlation between changes in the hub path radius and shaft deflection, a method for simulating the deflection response for a given input was determined. As seen earlier, Milne and Davis developed their method using a fixed radius and point of rotation, and constant physical properties along the length of the shaft. I developed this model by closely following a model developed by Brylawski [6]. Her model considered the club deflection in three dimensions. I have constrained my model to a single plane. Additionally, the physical properties of the shaft and the solution to the model differed significantly from that which she presented.

A moving coordinate system is defined, as shown in Fig. (4), with the $\mathrm{z}$ direction along the length of the shaft, the $\mathrm{x}$-direction perpendicular to the shaft and parallel to the club face, and the y-direction perpendicular to the shaft and club face. The $y$ and $\mathrm{z}$ axes at address define the plane of the swing and the properties of the shaft are dependent on the position $\mathrm{z}$.

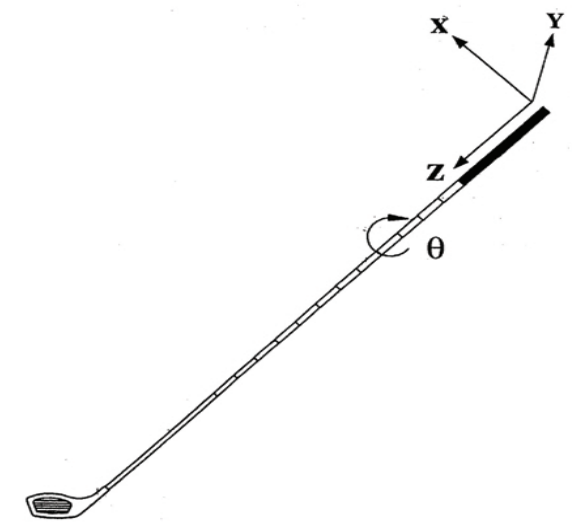

Fig. (4). Coordinate system defined for deflection model [6].

The equations of motion were developed using Hamilton's Principle which says that the sum of the variation in kinetic energy and virtual work of the system over a time interval from $t_{1}$ to $t_{2}$ is equal to zero (Equation 1).

$\int_{t_{1}}^{t_{2}}\left(\delta T-\delta V+\delta W_{n c}\right) d t=0$

The virtual work can be expressed as the nonconservative virtual work $\left(W_{n c}\right)$ minus the potential energy $(V)$ of the system. Where the non-conservative virtual work is a function of the applied forces and moments at the ends of the shaft and the distributed forces along its length and the potential energy is given by the elastic strain energy of the shaft. The equations of motion are derived by combining the total variation expressions for kinetic and potential energy and non-conservative virtual work as given by Hamilton's principle. Once the terms have been summed and the integral has been evaluated, we can group the terms based on variation. We know that the variation in $\mathrm{x}, \mathrm{y}$, theta, the partial derivative of $\mathrm{x}$ with respect to $\mathrm{y}$, and the partial of $\mathrm{y}$ with respect to $\mathrm{z}$ are arbitrary over the length of the shaft, so to obtain a reasonable solution, the coefficients of these terms must equal zero. Also, a property of variation is that all of these terms must equal zero at the end points of the shaft.

This development gives us the partial differential equation and boundary conditions for the shaft in all directions without a damping term. However, in order for a reasonable solution to be attained, a damping term needs to be added to the deflection equation so that the final equation is of the form displayed in Equation 2. The damping term represents the natural damping that is provided by the shaft material and the hands of the golfer. In forming the final equation, the distributed forces applied to the shaft are neglected because they are small compared to forces acting at the ends of shaft. This simplification allows for an initial solution to be attained, the reasonableness of which can be assessed by comparing the deflections at the club head to existing data. The equation of motion of the flexible club system, displayed in equation 2 , is driven by the forces in the $\mathrm{y}$ and $\mathrm{z}$ directions and the moment in the $\mathrm{x}$ direction applied to the grip end of the club by the golfer. The solution to the equation of motion gives the deflection of the club at every point along the shaft and at every time during the downswing.

$m_{z} \frac{\partial^{2} y(z, t)}{\partial t^{2}}+c \frac{\partial y(z, t)}{\partial t}+\frac{\partial^{2}}{\partial z^{2}}\left[E I_{A}(z) \frac{\partial^{2} y(z, t)}{\partial z^{2}}\right]=0$

The boundary conditions are applied at the grip and club head sides of the shaft, where $\mathrm{z}$ equals zero and $\mathrm{L}$ respectively. I made the choice to represent the grip side as a cantilever beam (Equations 3 and 4), because very little is known about the interactions between the golfer's hands and the club and this assumption makes the equations of motion easier to solve. This means that the deflection and slope of the shaft at the hands $(\mathrm{z}=0)$ are both equal to zero.

$$
\begin{gathered}
y(0, t)=0 \\
\frac{\partial y(0, t)}{\partial z}=0
\end{gathered}
$$

The final two boundary conditions are applied at the club head end of the shaft. This end is modeled as free and is subject to the conservation of force and moment equations shown in Equations 5 and 6 respectively.

$$
\begin{aligned}
& \frac{\partial}{\partial z}\left[E I_{A}(L) \frac{\partial^{2} y(L, t)}{\partial z^{2}}\right]+F_{y}(t)=0 \\
& -E I_{A}(L) \frac{\partial^{2} y(L, t)}{\partial z^{2}}-F_{z}(t) \frac{\partial y(L, t)}{\partial z}+M_{x}(t)=0
\end{aligned}
$$

For a solution to be obtained, the initial position and speed of the shaft need to be specified. For this investigation, I have assumed that the shaft is both undeflected and at rest at the initiation of the downswing (Equations 7 and 8 respectively).

$$
\begin{aligned}
& y(z, 0)=0 \\
& \frac{\partial y}{\partial t}(z, 0)=0
\end{aligned}
$$

Attention is focused on the deflection in the y-direction of the club coordinate system. This is where the majority of 
the deflection occurs and where deflection has the possibility of directly contributing to club head velocity and dynamic loft at impact. Also, existing data is available to drive the model in this direction. Force and moment information is missing if the model is to be driven to predict deflections in the $\mathrm{x}$ or theta directions.

\section{Motion Capture}

Data to kinematically drive the kinetic model are obtained from subject golf swings. A motion capture system (Motion Analysis Corporation, Santa Rosa, CA, USA) with eight Falcon HR 240 cameras and Eva 6.02 software is used to collect and process data from the golfers' swings. The system tracks passive-reflective markers that are placed on the golfer and the club. Only the data from the markers on the club are necessary to drive this model. The three markers on the club are arranged in a rigid triad that is attached to the shaft just below the handgrip. Two of the markers are aligned with the long axis of the club shaft, and the third is offset perpendicular to the club shaft and parallel to the club face. The three-dimensional marker triad paths are recorded at $200 \mathrm{~Hz}$ then smoothed and processed to yield global body 1-2-3 angular motions of the club and the three-dimensional global positions of the hub path using methods described in [7]. The orientation of the swing plane ( $X-Y$ plane) during the downswing is established from the angular motion data [8]. The global position data of the hub path and global orientation of the club are mapped onto this plane using methods described in [9] to yield the $X$ and $Y$ position data of the hub path and the swing angle of the club within the swing plane.

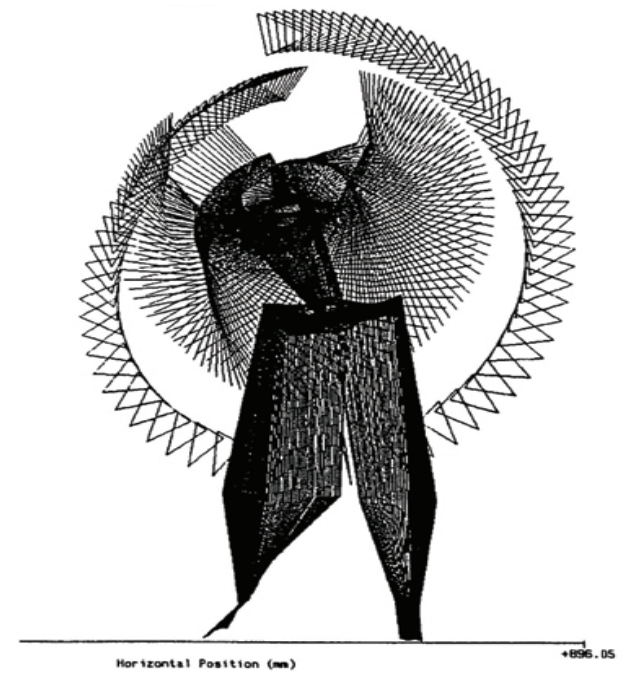

Fig. (5). Motion Capture Data.

Numerical differentiation of the swing plane linear and angular position data yields the linear acceleration of the hub path, and angular velocity and acceleration of the club [10].

\section{Subjects}

Three male amateur golfers had their golf swings analyzed for this study. All subjects are right-handed and their relevant data are given in Table 1. A diversity of skill levels and swing styles was the criteria for selecting these subjects in an attempt to yield a range of results. Stylistically, the scratch and five handicap golfers had aggressive, powerful, and quick swings, whereas the thirteen handicap golfer had a smoother, longer, and slower swing. All subjects used the same club (driver of length $=1.092 \mathrm{~m}$; mass $=0.382$ $\mathrm{kg}$; cg location from top of club $=0.661 \mathrm{~m} ; \mathrm{I}_{\mathrm{CG}}=0.07104$ $\mathrm{kg} \cdot \mathrm{m}^{2}$ ). Informed consent for the following procedure was obtained from all subjects. Each subject had reflective markers placed upon their body. A rigid triad of markers was attached to the club near the top of the shaft. The Motion Analysis system was calibrated until the combined 3D residual for all cameras was under $1.00 \mathrm{~mm}$. (Test/retest of static marker locations varied by less than $0.20 \mathrm{~mm}$ for a given calibration.) The subjects were asked to execute a series of swings that included hitting a ball into a net. The subjects were advised to swing the club in a manner similar to hitting a driver in a competitive situation where distance and accuracy were both important. The subjects were instructed to practice swinging the club as many times as necessary until they became comfortable with the testing situation and felt they could swing "normally" and consistently. Subsequently, several swings from each subject were recorded and tracked then presented to the subjects for their review. It was found that the club head velocities were consistent among the acceptable trials within a maximum range of $5 \%$ for all subjects. The subjects each selected what they considered to be their most representative swing in terms of club head velocity, impact feel, partial flight of the ball, and overall visual assessment of the motion capture data. This single self-selected swing from each subject was then analyzed for this study.

\section{Solution to Deflection Model}

It was necessary to determine a method for solving the equations so as to generate meaningful deflection data for each golfer's swing. The equation is a fourth order partial differential equation with non-constant coefficients. To evaluate the form of the differential equation, its discriminant needs to be determined. A discriminant of less than zero yields an elliptic equation, equal to zero a parabolic equation, and greater than zero a hyperbolic equation [11]. The equation of motion considered here spends the majority of its solution domain space in the hyperbolic form.

A numerical solution method is employed in order to find a solution to the differential equation. Mathematica provides

Table 1. Subject Data

\begin{tabular}{|c|c|c|c|c|c|c|}
\hline Subject & Age & Height $(\mathbf{c m})$ & Weight $(\mathbf{k g})$ & Handicap & Experience (Years) & Round per Year \\
\hline \hline 1 (Male) & 42 & 183 & 86.3 & 0 (scratch) & 24 & 150 \\
\hline 2 (Male) & 35 & 179 & 93.1 & 5 & 13 & 7 \\
\hline 3 (Male) & 21 & 188 & 74.9 & 100 & 120 \\
\hline
\end{tabular}


a numerical solver that can handle all three forms of partial differential equations. The solver, NDSolve, utilizes the numerical method of lines to find a solution to partial differential equations with sufficient boundary conditions. This method discretizes the equation in all but one direction and then integrates the newly formed system of ordinary differential or differential algebraic equations to obtain a solution. The output, or solution, produced by the NDSolve command is a two dimensional interpolating function that, in this case, gives y deflection data based on position along the shaft (z), and time (t) [12]. A three-dimensional plot of the interpolating function produced for the scratch golfer is shown in Fig. (6).

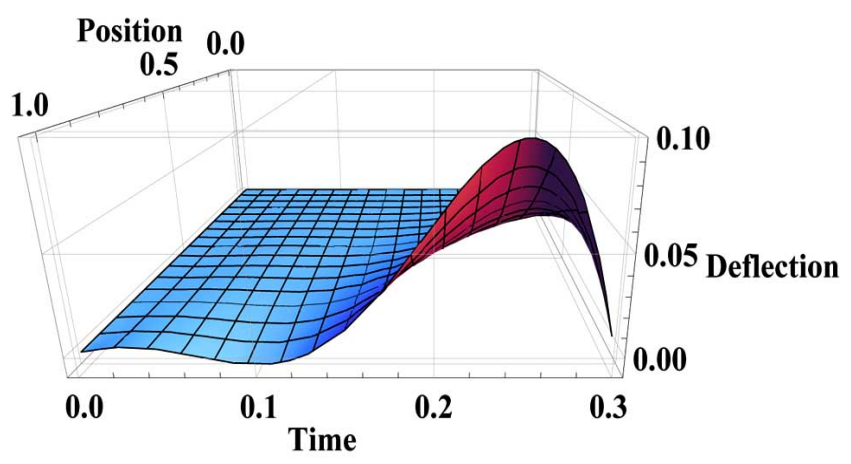

Fig. (6). 3-D plot of club shape during downswing for scratch golfer.

The physical properties of the model are the mass per unit length for the golf club $\left(\mathrm{m}_{\mathrm{z}}\right)$ and the effective stiffness of the shaft (EI). The stiffness value is dependent on the modulus of the shaft material as well as the shaft's second moment of area. Since a golf shaft is often stepped, the second moment of area varies along its length. The varying stiffness along the length of the shaft is displayed in Fig. (7) as the non-continuous horizontal lines.

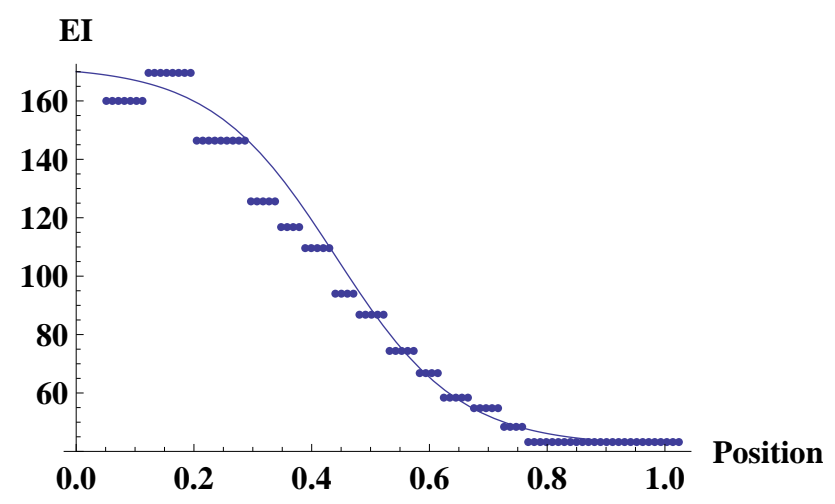

Fig. (7). Curve fit of equation to EI data.

Because the stiffness values are non-continuous, there are stability issues when attempting to solve the differential equation, even when heavily damped. To solve this problem, a curve was fit to the data that approximates the stepped nature of the shaft while maintaining its variability in stiffness along the length. The equation for the curve fit is shown in Equation 9.

$$
E I=\frac{65}{1+e^{10(z-0.41)}}+21
$$

The final parameter that needs to be defined is the damping (c) applied to the system by the hands of the golfer and the shaft material. An iterative approach was used to determine $\mathrm{c}$ as follows: An initial solution to the model was found, and then the value of $\mathrm{c}$ was altered until the club head deflection data determined from the model matched published data for the three golfers that were investigated. In most cases, a very close fit was obtained between actual deflection and that predicted by the model (see Fig. 8).

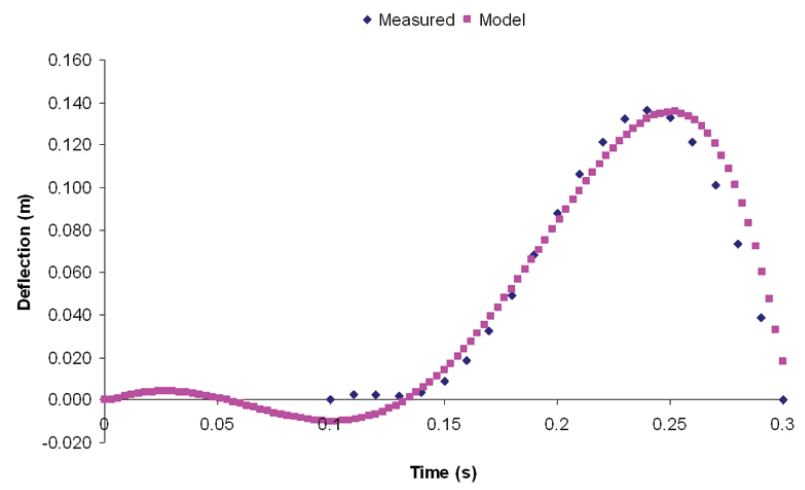

Fig. (8). Deflection of club head during downswing, measured vs. model.

The oscillating deflection progression of the club head is consistent with published results $[2,3,5]$. The shape of the club shaft at impact is also a very important part of the information that can be extracted from the solution of the equation of motion as it determines the dynamic loft added to the club face. The deformed shape of the club at impact for the scratch golfer is shown in Fig. (9).

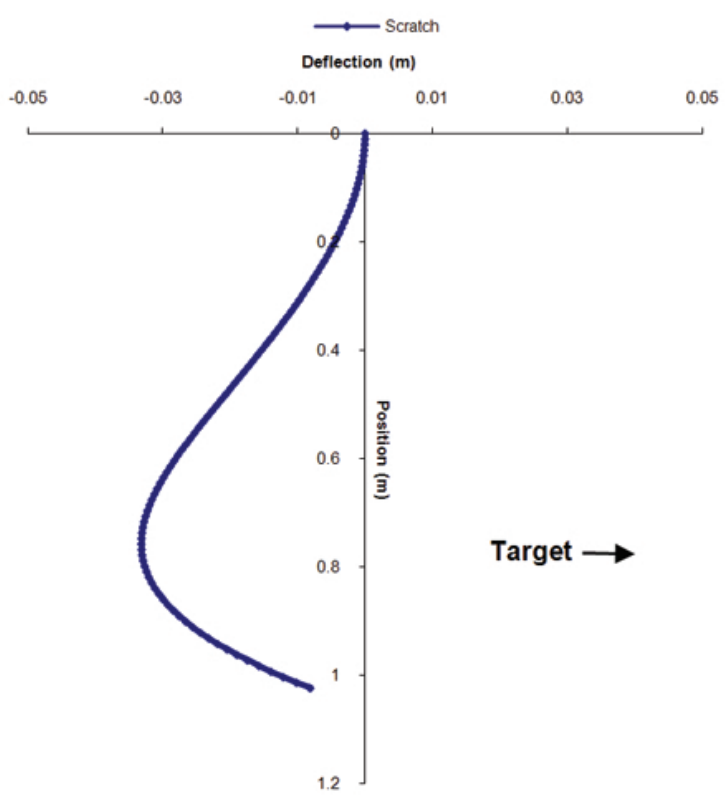

Fig. (9). Deformed shape of club for scratch golfer at impact. 

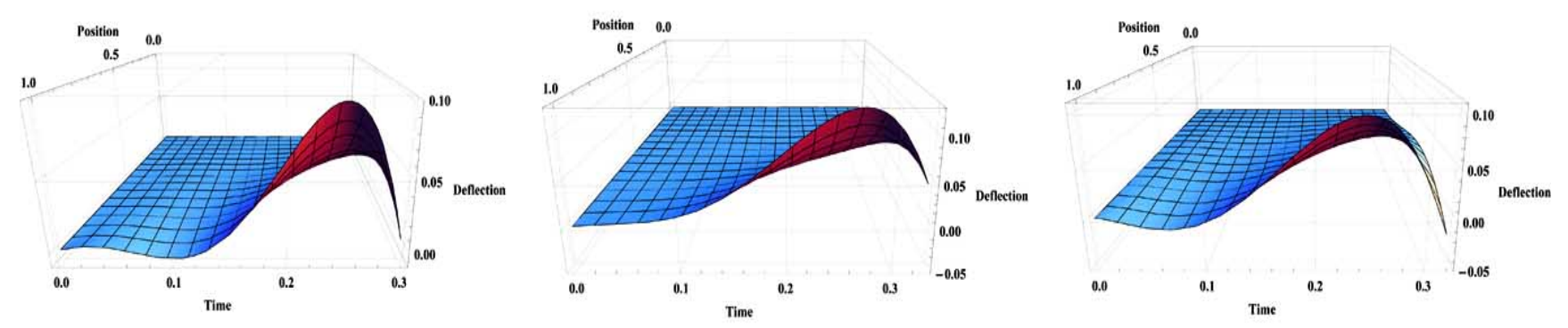

Fig. (10). Deflection progression for each of the three subjects tested (Scratch - left, $5 \mathrm{H}$ - middle, 13H - right), all exhibit first bending mode at point of maximum club head deflection which quickly moves to the second bending mode at impact.

This impact shape for the club is also supported by the literature $[2,3,5]$. The source of added dynamic loft at impact that is discussed in Milne \& Davis [2] can clearly be seen in this figure as the convex portion of the curve is faced in the direction of motion of the club.

\section{RESULTS AND DISCUSSION}

The swing data for the three subjects was analyzed using the rigid body model to determine the kinetic inputs from the golfer to the club. These kinetic inputs were applied to the deflection model and then the model was solved. Several iterations were necessary to reach convergence and to identify an appropriate value of club damping. Fig. (10) illustrates the deflection behavior of the golf club during the downswing for the three subjects. Time is from the initiation of the downswing until impact $(t=0)$. Deflection is in only the plane of the swing (y-direction). Position is the distance in meters from the grip point of the shaft $(\mathrm{z}=0)$.

As can be seen in Fig. (10) the shaft for each golfer displays a bending progression during the downswing which cycles through the first two bending modes. (The first bending mode is a classical cantilever beam bending mode while the second is a characteristic bending mode for a beam with cantilever and pin supports. This is described later in this section) This mode one to mode two progression is most pronounced during the time just before impact where the club head deflection reaches its maximum and then moves back towards its un-deflected position near impact.

It is important to investigate the reasons behind the deflection progression during the downswing because it has a direct influence on the golf shot. To aid in this investigation, we will consider the downswing as being broken into three phases as outlined by [1]. The phases are outlined by maximums in the hub path radius. The most integral portion of the deflection is the time just before impact, phase three, where the mode one to mode two bending progression is most pronounced. This is the point in the swing where shaft flexibility adds velocity and dynamic loft to the club head. In Figs. (11 and 12) below, the scratch golfer's club head deflection is superimposed on plots of his hub path radius and the interaction torque respectively.

The first phase of the downswing shows a local minimum in club head deflection coupled with a local maximum in hub path radius. During this phase, there is a reduction in radius while the club head reaches $42 \%$ of its maximum deflection and the torque reaches $80 \%$ of its maximum value. During the phase, torque also drops to its minimum value $3 \mathrm{~ms}$ before the deflection does the same.

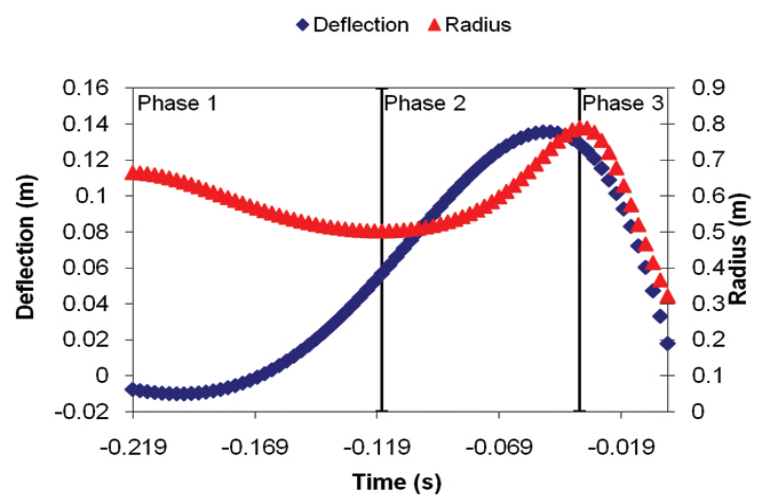

Fig. (11). Club head deflection and hub path radius for scratch golfer.

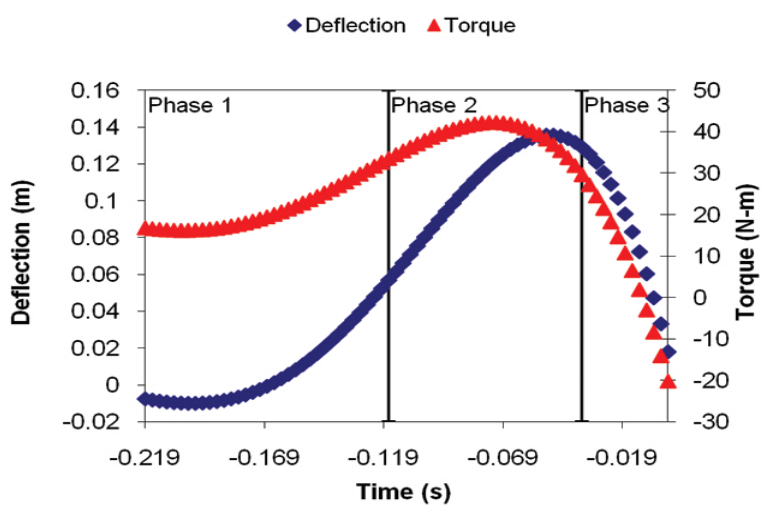

Fig. (12). Club head deflection and applied torque during downswing for scratch golfer.

The second phase of the downswing shows the radius increasing from a local minimum to its absolute maximum at the beginning of phase three. The torque and club head deflection curves also reach their respective maximums during phase two, with the torque reaching its value $24 \mathrm{~ms}$ before the deflection. As shown in Fig. (12) above, there is clearly a relationship between swing torque and club head deflection. The curves of their behavior during the downswing have very similar shapes, with the maximum and minimum values of the torque curve occurring just before those of the club 
head deflection. It is known that hub path has a significant effect on the timing of the changes in the torque applied to the club [1], and from Figs. (11 and 12), it appears that the torque has the same effect on club head deflection. Additionally, the delay between changes in torque and changes in club head deflection can be attributed to the time it takes for affects to propagate down the shaft.

Phase three is characterized by a drastic reduction in hub path radius as well as club head deflection and the applied torque. With respect to the kinetic inputs to the club specifically, the torque decreases from an absolute maximum to nearly zero, the tangential force drops because the club has reached maximum velocity in that direction, and the normal force increases significantly. This occurs in an effort to harvest the last remaining energy in the system, converting it into club head velocity at impact. Assuming that, when compared to the normal force, tangential force and torque are virtually negligible during this period, it is logical that a large normal force would result in a significant reduction in club head deflection. This behavior is due to the offset of the center of mass of the club head behind the centerline of the shaft. This geometry will produce a resultant moment that acts on the club head in response to a large normal force applied to the grip. This moment will work to negate any deflection in the club head while producing the shaft shape that we see at impact. The change in shaft shape as well as the applied normal force $\left(\mathrm{F}_{\mathrm{N}}\right)$ and resultant moment $\left(\mathrm{M}_{\mathrm{R}}\right)$ acting on the club head are shown in Fig. (13) below.

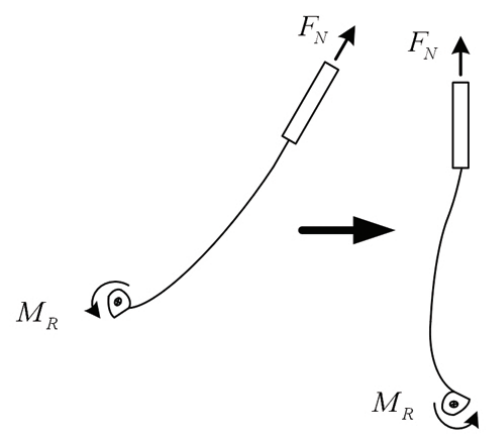

Fig. (13). Affect of increased normal force on shape of club (deflections greatly magnified).

This analysis proves the existence of two main relationships. The first is that during all three phases of the downswing, the torque applied to the club has a definite impact on club head deflection. The shape of the club head deflection and torque curves are very similar and reach respective minima and maxima within several milliseconds of each other. The torque curve reaches its maxima and minima before the deflection curve which physically makes sense. The second relationship, the rebounding of the club just before impact, is a result of the drastic increase in normal force. The timing of both of these behaviors is dependent on hub path radius [1]. This means that hub path radius is integral in determining the timing of shaft deflection which has a considerable affect on club head velocity and dynamic loft of the club face at impact.

\section{SHAFT FITTING METHODOLOGY}

This information regarding hub path's affect on shaft behavior is all motivation to develop a shaft fitting method that uses golfer-specific club behavior to determine the shaft stiffness that will maximize carry distance of the golf ball. To do this, the position of the flexible golf club needs to be determined at every point during the downswing. Having completed this by developing a flexible golf club system equation of motion and solving it, the next step is to run a variety of shaft stiffness values through the deflection model for a specific golfer's kinetic inputs to the club. Then, the shaft that produces the longest theoretical carry distance based on input from both the deflection of the club head during the downswing and the shape of the entire shaft at impact is the ideal shaft for that golfer.

To select appropriate shaft stiffness values it was necessary to characterize the absolute stiffness of a shaft. Frequency testing is one way to characterize the stiffness of a shaft [13]. Existing literature shows that the frequency that produces the first bending mode of a shaft varies from about $5 \mathrm{~Hz}$ for an extra stiff shaft to about $3 \mathrm{~Hz}$ for an extra flexible shaft $[2,13,14]$.

For this model, eight shaft stiffness values were investigated. The geometry of the shaft was held constant while the modulus value was changed to approximate the effect of using different shaft stiffnesses. Finite element methods (Ansys) were used to determine the modes and frequencies of a number of different shaft configurations. The first three bending modes for a characteristic golf shaft are displayed in Fig. (14).

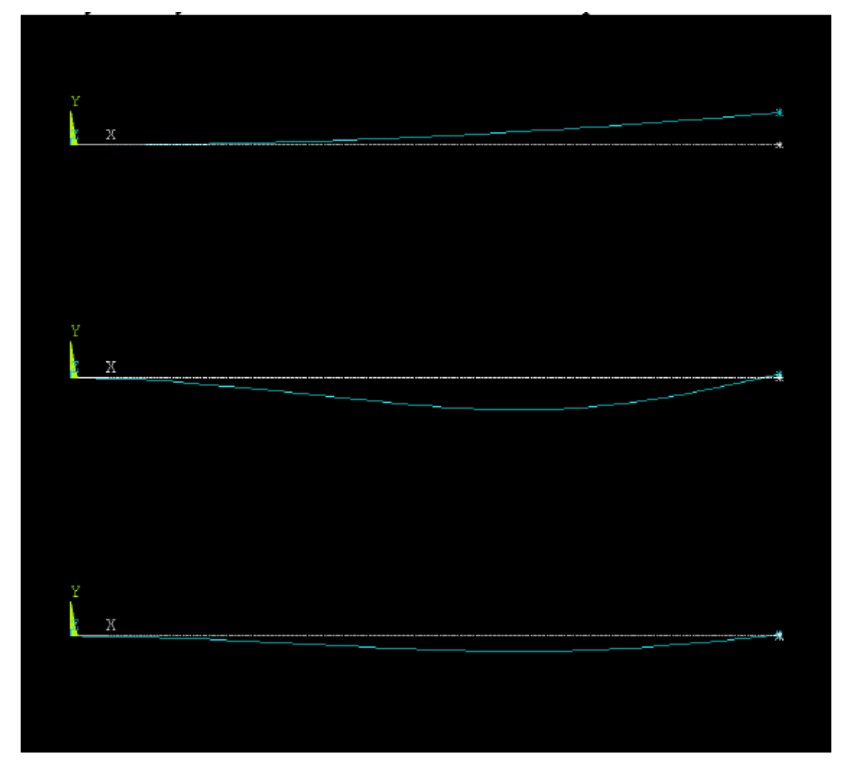

Fig. (14). The deflection shape of the first three bending modes as predicted by the finite element model in Ansys.

As you can see in Fig. (14), the first bending mode is the typical cantilever beam bending mode while the second is that for a beam with cantilever and pin supports. The frequency data for the eight shafts that were tested are shown in Table 2.

As can be seen from the table, the frequencies and their corresponding shaft stiffness, span the range of published data. The most flexible shaft has a bending frequency that is two-thirds of the minimum published value and the stiffest shaft has one that is twice the highest published value. 
Table 2. Frequency of $1^{\text {st }}, 2^{\text {nd }}$, and $3^{\text {rd }}$ Bending Modes of Shafts Considered in Fitting Scheme

\begin{tabular}{|c|c|c|c|}
\hline Stiffness & \multicolumn{3}{|c|}{ Frequency (Hz) } \\
\hline$\left(*^{\left.* \mathbf{1 0}^{\mathbf{1 1}}\right)}\right.$ & $\boldsymbol{1}$ & $\mathbf{2}$ & $\mathbf{3}$ \\
\hline \hline 0.5 & 2.180 & 23.403 & 71.941 \\
\hline 1 & 3.083 & 33.097 & 101.740 \\
\hline 2 & 4.360 & 46.806 & 143.880 \\
\hline 3 & 5.340 & 57.326 & 176.220 \\
\hline 4 & 6.166 & 66.194 & 203.480 \\
\hline 5 & 6.894 & 74.007 & 227.500 \\
\hline 6 & 7.552 & 81.071 & 249.210 \\
\hline 7 & 8.1566 & 87.567 & 269.18 \\
\hline
\end{tabular}

Looking purely at club head velocity at impact, a flexible shaft yields a larger velocity than a rigid one [5]. It would follow then that every golfer should use a very flexible shaft. However, if you refer back to Fig. (9) above, which depicts the shape of the scratch golfer's club at impact, you can see that a flexible shaft causes a change in orientation of the clubface with respect to the ball. Effectively, the deformed shaft adds more loft. This turns shaft fitting into an optimization problem where the added loft needs to be balanced with increases in club head velocity.

The output of the deflection model predicted the deflected position of the club head, the shape of the shaft at impact (dynamic loft), and the velocity of the club head throughout the downswing. Using an assumed value for the coefficient of restitution of the ball (e) of 0.78 [15], and assumed club head $\left(\mathrm{m}_{\mathrm{ch}}\right)$ and ball $\left(\mathrm{m}_{\mathrm{b}}\right)$ masses of $205 \mathrm{~g}$ and $46 \mathrm{~g}$ respectively, then the velocity of the ball after impact can be determined through conservation of momentum.

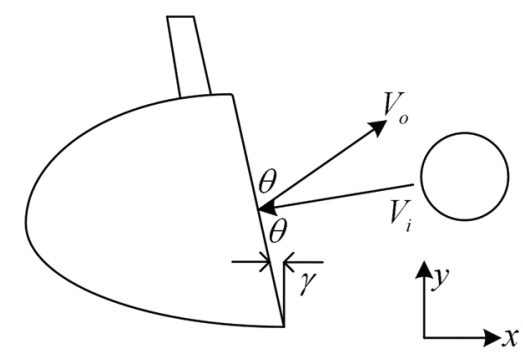

Fig. (15). Ball hitting club head and bouncing.

The post impact velocity of the ball $\left(\mathrm{V}_{\mathrm{o}}\right)$, hang time $\left(\mathrm{t}_{\mathrm{h}}\right)$, and carry distance $\left(\mathrm{s}_{\mathrm{x}}\right)$ are determined by Equations 10 through 14 below:
$V_{o}=\frac{m_{c h} \cdot V_{c h, i}(1+e)}{m_{b}+m_{c h}}$

$V_{o x}=V_{o} \sin (\theta-\gamma)$

$V_{o y}=V_{o} \cos (\theta-\gamma)$

$t_{h}=\frac{2 V_{o y}}{g}$

$s_{x}=V_{o x} \cdot t_{h}$

Where, $\mathrm{V}_{\text {ch,i }}$ is the pre-impact velocity of the club head, $\theta$ is the angle between the pre-impact velocity vector and the clubface, $\gamma$ is the total dynamic loft of the clubface (loft of club plus loft added by shaft deflection), and $\mathrm{g}$ is the acceleration of the ball due to gravity.

\section{Shaft Fitting}

Table 3 shows the results of the shaft fitting model. Each value is the percentage of the maximum carry distance predicted for that particular golfer. This means that the shaft stiffness that corresponds to 100.0 in the table produced the longest carry distance.

Table 4 presents the dynamic loft $(\gamma)$, impact velocity components $\left(\mathrm{V}_{\mathrm{ix}}, \mathrm{V}_{\mathrm{iy}}\right.$, and $\left.\mathrm{V}_{\mathrm{i}}\right)$, impact angle $(\theta)$, and carry distance $\left(S_{\mathrm{x}}\right)$ for each subject using the ideal shaft.

Table 4. Dynamic Loft, Velocity Components, Impact Angle, and Carry Distance of Fitted Shafts for Three Golfers

\begin{tabular}{|c|c|c|c|}
\hline Parameter & Scratch & $\mathbf{5 H}$ & $\mathbf{1 3 H}$ \\
\hline \hline Dyn. Loft (deg) & 21.03 & 19.85 & 21.89 \\
\hline $\mathrm{V}_{\text {ox }}(\mathrm{m} / \mathrm{s})$ & 55.83 & 50.37 & 47.30 \\
\hline $\mathrm{V}_{\text {oy }}(\mathrm{m} / \mathrm{s})$ & 57.99 & 52.32 & 51.60 \\
\hline $\mathrm{V}_{\text {o }}(\mathrm{m} / \mathrm{s})$ & 80.49 & 72.63 & 70.00 \\
\hline Imp. Angle (deg) & 64.94 & 63.77 & 64.40 \\
\hline $\mathrm{S}_{\mathrm{x}}(\mathrm{m})$ & 660.01 & 537.28 & 497.66 \\
\hline
\end{tabular}

The club fitting model yielded significant results. The three golfers analyzed, all of whom had significantly different swing styles, yielded different ideal shaft flexibilities. Stylistically, the scratch and five handicap $(5 \mathrm{H})$ golfers had aggressive, powerful, and quick swings, whereas the 13 handicap golfer $(13 \mathrm{H})$ had a smoother, longer, and slower swing.

Table 3. Results of Shaft Fitting Model - Percent of Maximum Ball Carry for Differing Shaft Stiffness

\begin{tabular}{|c|c|c|c|c|c|c|c|c|}
\hline $\mathbf{E I} \mathbf{1 0}^{-\mathbf{1 1}}$ & $\mathbf{0 . 5}$ & $\mathbf{1}$ & $\mathbf{2}$ & $\mathbf{3}$ & $\mathbf{4}$ & $\mathbf{5}$ & $\mathbf{6}$ & $\mathbf{7}$ \\
\hline \hline Scratch & -53.1 & 25.8 & 86.3 & 98.8 & $\mathbf{1 0 0 . 0}$ & 98.6 & 89.8 & 87.2 \\
\hline $5 \mathrm{H}$ & 98.0 & $\mathbf{1 0 0 . 0}$ & 93.7 & 87.8 & 83.9 & 81.3 & 79.6 & 78.3 \\
\hline $13 \mathrm{H}$ & 27.0 & 70.3 & 96.3 & $\mathbf{1 0 0 . 0}$ & 99.4 & 98.0 & 96.4 & 94.9 \\
\hline
\end{tabular}


Referring to Table 4, it seems that all of the optimum shafts provided a dynamic loft of around $20^{\circ}$ and an impact angle around $64^{\circ}$. These could possibly be optimum values across the population of golfers, but more tests need to be conducted to verify this claim beyond a level of reasonable doubt. Additional support for this idea of common optimum shaft characteristics comes from Fig. (16) below, which depicts the deformed shape of each of the optimized clubs at impact.

As you can see, all three shafts exhibit the same bending mode at impact. Based on this information, it seems that

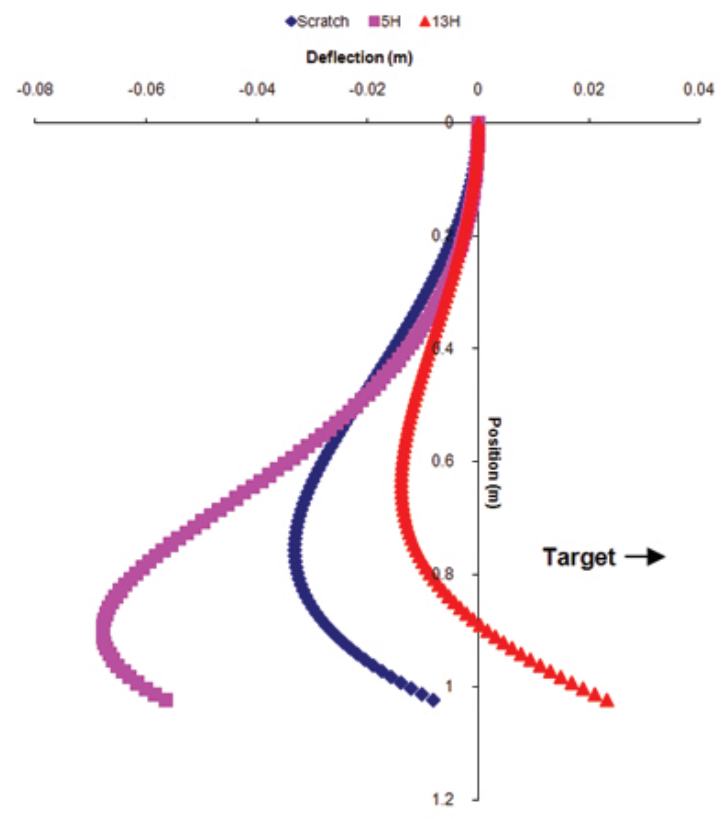

Fig. (16). Deflection at Impact.

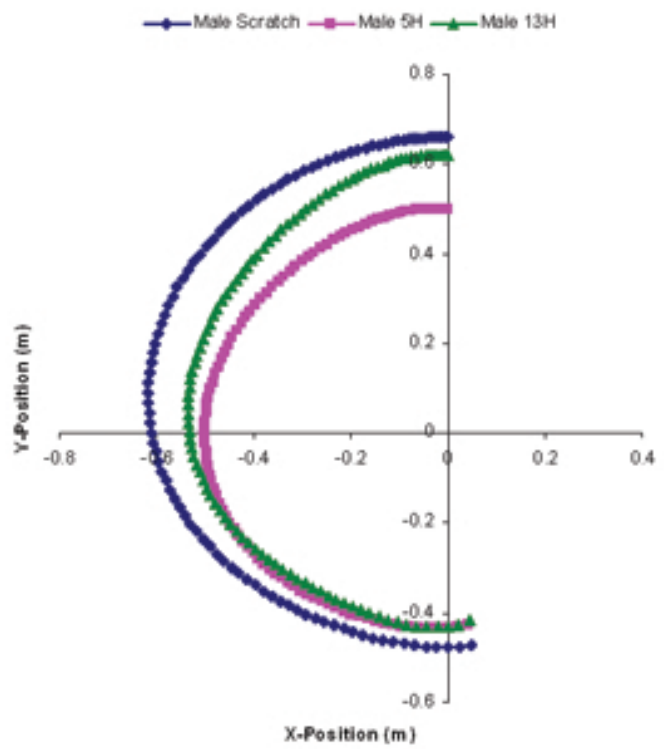

shaft fitting is an exercise in finding the shaft that, for a subject's torque and force inputs, will produce a deformed shape like the ones in Fig. (16) along with an impact angle of around

Through observation it is known that the scratch and five handicap golfers have similar aggressive swings. Conventional golf wisdom dictates that a golfer with an aggressive swing should use a stiffer shaft. However, the fitting model predicts that the five handicap golfer should use the most flexible shaft of the three golfers tested. I believe that this selection is made clear by observing the hub path and its radius during the downswing of each of the three golfers as shown in Fig. (17).

When considering Fig. (17 a) above, both the scratch and 13 handicap golfers have fairly large hub paths when compared to the five handicap golfer who exhibits a uniquely compact swing hub. Based on the hub path radius plot in Fig. (17 b), it is clear that the scratch and 13 handicap golfers experience much larger changes in radius than the five handicap golfer. Additionally, when considering the third phase of the downswing specifically, the scratch and 13 handicap golfers decrease their radius values by 60 and $85 \%$ respectively, while the five handicap golfer only decreases his by $42 \%$. These more drastic changes in radius seem to necessitate a stiffer shaft to ensure that the club head experiences its return to the ideal dynamic loft and impact angle before striking the ball.

\section{Limitations of Study}

While the results of the study compared well with published data, the reader should keep in mind the context and limitations of the study. The model restricted motion to a single plane and deflections in the $\mathrm{z}$ direction were ignored. In addition, the results and discussions were based upon three subjects, one swing for each subject. 


\section{CONCLUSIONS}

This thesis presented an investigation of the relationships between hand path and club head deflection during the downswing portion of the golf swing. Using an equation of motion that described club behavior in the plane of the swing it was determined that hub path has a significant, though secondary, effect on shaft deflection behavior. Specifically, hub path affected the resulting club head orientation including the timing of club head deflection, and club head velocity at impact. From these findings a shaft fitting method was proposed that uses predicted shaft deflections, dynamic loft, and club head velocity to select an ideal shaft stiffness profile that maximizes carry distance of a golf ball for a given subject.

\section{REFERENCES}

[1] Nesbit SM, McGinnis R. Analysis of the swing hub of the golf shot. J Sports Sci Med 2009; 8(2): 235-46.

[2] Milne RD, Davis JP. The role of the shaft in the golf swing. J Bio 1992; 25(9): 975-83.

[3] Mather JSB, Jowett S. Three-dimensional shape of the golf club during the swing. Eng Sport 2000; 77(10): 77-85.

[4] Cochran A, Stobbs J. The search for the perfect swing. New York: J.P. Lippincott Co. 1969.
[5] Penner R. The physics of golf. Rep Prog Phys 2002; 66: 131-71.

[6] Brylawski AM. A Numerical Model for Determining the ThreeDimensional Deformations of a Golf Shaft during the Swing. Masters Thesis, Lehigh University 1994.

[7] Nesbit SM. A three-dimensional kinematic and kinetic study of the golf swing. J Sports Sci Med 2005; 4: 499-519.

[8] Coleman S, Rankin A. A three-dimensional examination of the planar nature of the golf swing. J Sports Sci 2005; 25: 227-34.

[9] Kane TR, Likins PW, Levinson DA. Spacecraft dynamics. New York: McGraw-Hill Co. 1983.

[10] Dean RK, Nesbit SM. Evaluation of finite difference schemes for the solution of the inverse velocity and acceleration problem for robot manipulators. Proceedings of the Third International Conference on CAD/CAM, Robotics, and Factories of the Future; 1988: Southfield, Michigan: edited by Brendra Prasad 1988: pp. 210-6.

[11] O'Neil PV. Beginning partial differential equations. USA: Wiley 1999.

[12] Wolfram S. Numerical Solution of Partial Differential Equations. Mathematica Tutorial 2006.

[13] Cheong SK, Kang KW, Jeong SK. Evaluation of the mechanical performance of golf shafts. Eng Fail Anal 2006; 13(3): 464-73.

[14] Miao T, Watari M, Kawaguchi M, Ikeda MA. study of clubhead speed as a function of grip speed for a variety of shaft flexibility, Proceedings of the 1998 World Scientific Congress of Golf: St. Andrews, Scotland 1998.

[15] Chou PC, Liang D, Yang J. Contact forces, coefficient of restitution, and spin rate of golf ball impact. Proceedings of the 1994 World Scientific Congress of Golf: St. Andrews, Scotland 1994. 